\title{
Mutual emotional understanding in a face-to-face communication environment: How speakers understand and react to listeners' emotion in a game task dialog
}

\author{
Yoshiko Arimoto $^{1,2, *}$ and Kazuo Okanoya ${ }^{1,2}$ \\ ${ }^{1}$ Graduate School of Arts and Science, the University of Tokyo, 3-8-1 Komaba, Meguro-ku, Tokyo, 153-8902 Japan \\ ${ }^{2} J S T$, ERATO Okanoya Emotional Information Project
}

(Received 2 October 2014, Accepted for publication 19 January 2015)

Keywords: Emotional understanding, Emotional synchrony, Dyadic dialog, Subjective evaluation PACS number: 43.71.Bp [doi:10.1250/ast.36.370]

\section{Introduction}

Speakers continue their dialog by coordinating their utterances and actions to share a mutual belief, or a common ground, regarding their knowledge of themselves to achieve their task [1]. In other words, the speakers mutually and implicitly believe that they share a common ground regarding what the other person thinks and feels during the dialog. However, they never explicitly confirm what they really share; thus, they may continue talking with and reacting to each other based on an uncertain (mutual?) belief. These mutual beliefs include the knowledge of an intention, an attitude, or an emotion of the interlocutors who participate in the dialog. The present study focuses on the mutual emotional understanding between speakers in a face-to-face communication environment to elucidate whether their beliefs of their partners' emotion modulated (or did not modulate) their emotion during the dialog.

First, the accuracy of the listener's understanding of his/her partner's emotion was investigated. Several attempts were made to investigate how accurately the listeners understood their partner's emotion, i.e., empathic accuracy [2-5]. The literature on the studies of empathic accuracy is briefly reviewed in [6]. According to Ickes et al. [2], listeners understand speakers' complex emotion (written descriptions of how they feel or think) with $21.7 \%$ accuracy $(S D=12.1)$ and understand speakers' valence (positive, neutral and negative) with $40.1 \%$ accuracy $(S D=17.1)$ throughout a dialog. However, these results were obtained by analyzing the speakers' viewpoints and did not account for the listeners' viewpoints. If the listeners' viewpoints were reflected in the analysis, the results might be different, and listeners' inaccurate understanding of the speakers' emotion would increase.

Then, the emotional synchrony between the interlocutors is investigated. Emotional synchrony, i.e., emotional contagion, is defined that the listener comes to feel the same emotion that the speaker feels [6-9]. Because emotional synchrony is based on the accurate emotional understanding of a partner [6], emotional synchrony is considered one of the phenomena of emotional understanding. If a listener does not understand his/her partner's emotion, emotional synchrony will not actually occur during their dialog. Therefore, the

*e-mail: ar@brain.riken.jp listener's emotion might be less synchronized with the speaker's actual emotion than is believed by the listener.

The present study investigated the following two questions: (1) how accurately a listener understands a speaker's emotion, and (2) whether believed emotional synchrony occurs more often than actual emotional synchrony due to the listener's inaccurate emotional understanding of the speaker.

\section{Material}

Fifty-two speakers (mean age $=21, S D=2.34$ ) participated in two types of 10-minute dyadic dialogs with a friend of the same sex. Thirteen pairs were female pairs, and the rest were male pairs.

The present study aimed to investigate the mutual emotional understanding that occurs between interlocutors during a spontaneous dialog. However, a critical issue noted with respect to naturalistic materials is that even a large speech corpus contains few emotional samples [10]. To solve this issue, a novel paradigm was proposed to record naturalistic expression that contains the spontaneous emotions of speakers using the games and its effectiveness was demonstrated [11,12]. The present study adopted the game task with reference to the paradigm of previous studies [11,12]. Using the game task, the speakers talked with their friends with more naturally expressed emotion than they did when using another task [11]. Adopting the game task in this study enabled us to investigate emotional understanding during a spontaneous dialog where the speakers did not hesitate to be emotional.

Each pair participated in two types of tasks: a competitive task and a cooperative task. The competitive task was a Japanese word-chain game, cap verses (Shiritori). The cooperative task was a simple game in which each pair worked together to raise their score to 100 . Because the pairs were not told what types of events raised the score, they had to discuss it and cooperate with each other to raise their score.

The speakers' behavior (speech and facial expressions) was recorded during the dialogs. Their speeches were recorded in individual channels of an audio stream with a sample rate of $48 \mathrm{kHz}$ and 16-bit precision. Each speaker's behavior was recorded at 30 frames per second on images of $640 \times 480$ resolutions with four CCD cameras. One of the cameras focused on the speaker's face. For synchronous audio 
Table 1 The total number of utterances and the mean number of utterances per speaker in each task. The standard deviations are indicated in the brackets.

\begin{tabular}{cccr}
\hline & Competitive & Cooperative & Total \\
\hline Numbers & 8,066 & 10,366 & 18,432 \\
Male & 3,985 & 5,311 & 9,296 \\
Female & 4,081 & 5,055 & 9,136 \\
Average & $155(53)$ & $199(39)$ & $354(80)$ \\
Male & $153(50)$ & $204(40)$ & $327(75)$ \\
Female & $157(56)$ & $194(39)$ & $351(86)$ \\
\hline
\end{tabular}

and visual recording, the audio-visual recording system Potato (Library Co. Ltd., Tokyo, Japan) was used.

Each speech signal was segmented based on 200-ms interpausal units (IPUs). The segmented utterances were orthographically transcribed into kanji (Chinese logograph) and kana (Japanese syllabary). The total number of utterances collected was 18,432 (8,066 in the competitive task and 10,366 in the cooperative task). Table 1 shows the total number of utterances and the mean number of utterances for each task. The mean number of utterances per speaker was $354(S D=80,155$ utterances $(S D=53)$ in the competitive task and 199 utterances $(S D=39)$ in the cooperative task).

\section{Dynamic emotional state evaluation}

The speakers also conducted subjective evaluations of their dynamic emotional state using GTrace [13]. The speakers dynamically rated their own emotional state (the speaker's self-evaluation) and their listener's emotional state (the listener's evaluation of the speaker's emotion) by watching the recorded audio-visual video sequences of the competitive task and cooperative task dialogs. They rated their own and the listener's emotional state based on a continuous emotional scale from -1 to 1 . The target emotional states were arousal $(\operatorname{aroused}(1)-$ sleepy $(-1))$, valence (pleasant(1)-unpleasant $(-1)$ ) and positivity (positive(1)-negative $(-1)$ ). The mean evaluated value of each emotional state was calculated at 10-second intervals as a measure of the dynamic emotional state of the speakers. Moreover, only the values in the mid-eight-minute period of the dialog were adopted for the following analysis after removing the values in the first and last 60 seconds of the dialog.

Figure 1 shows a chronic flow of the emotional state of each speaker of one pair during a competitive task dialog. It indicates that the emotional states of each speaker were consistent in arousal but inconsistent in valence. It suggests that one of the pairs felt pleasant but the other felt unpleasant because each speaker competed with each other during the dialog.

\section{Assessment of emotional understanding}

\subsection{Understanding speaker's emotion}

To measure emotional understanding with the emotional state evaluation, each pair's correlation coefficients between the listener's evaluation of the speaker's emotion and the speaker's self-evaluation of his/her emotion were calculated.
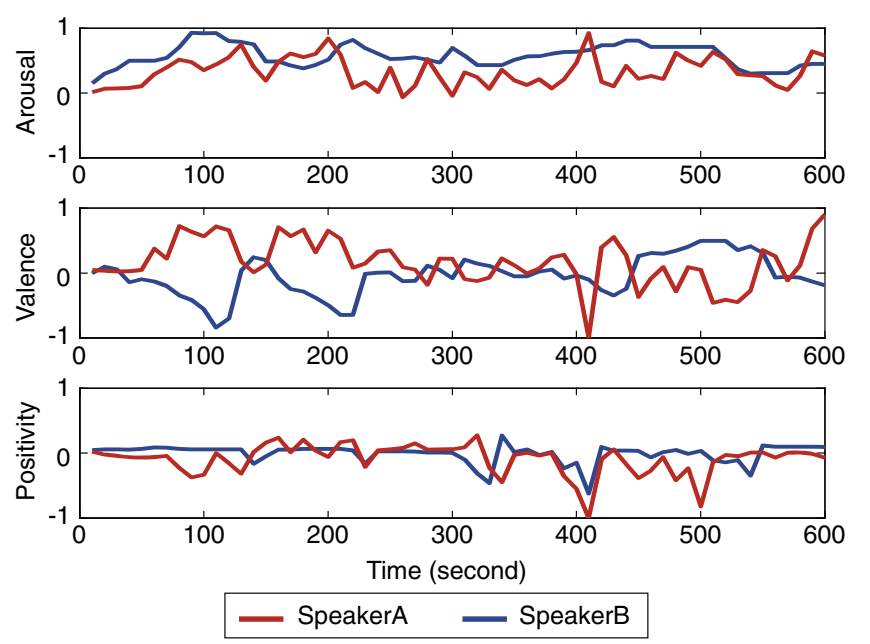

Fig. 1 Result of the dynamic emotional state annotation. Each panel shows the chronic flow of the emotional state of each speaker of one pair (for a competitive task).

Table 2 Number of listeners whose evaluations of speakers' emotion were significantly correlated with speakers' self-evaluation.

\begin{tabular}{llccc}
\hline \multirow{2}{*}{ Correlation } & Task & \multicolumn{3}{c}{ Emotion } \\
\cline { 3 - 5 } & & Arousal & Valence & Positive \\
\hline \multirow{2}{*}{ Pos. $(p<0.05)$} & Comp. & 27 & 33 & 14 \\
& Coop. & 19 & 31 & 18 \\
Neg. $(p<0.05)$ & Comp. & 3 & 3 & 4 \\
& Coop. & 3 & 1 & 4 \\
NoSig. & Comp. & 22 & 16 & 34 \\
& Coop. & 30 & 20 & 30 \\
\hline
\end{tabular}

The correlation coefficients indicate the accuracy of the listener's emotional understanding of the speaker. A positive correlation implies that the listener accurately understood the speaker's emotion through the dialog. On the contrary, a negative correlation implies that the listener understood the speaker's emotion as the opposite. No correlation implies that the listener did not understand the speaker's emotion at all.

The mean correlation coefficient of each emotional state during the competitive task was 0.22 for arousal, 0.28 for valence, and 0.14 for positivity. The mean correlation coefficient of each emotional state during the cooperative task was 0.17 for arousal, 0.30 for valence, and 0.16 for positivity. These results suggest that the listeners did not understand the speakers' emotion accurately in either task. Table 2 shows the numbers of listeners whose evaluation of the speakers' emotion were significantly correlated ("Pos." or "Neg." in Table 2) or not significantly correlated ("NoSig." in Table 2) with the speakers' self-evaluation of their own emotion. "Pos." and "Neg." indicate a positive correlation and a negative correlation, respectively. These results indicate that one half of the listeners understood their partners' arousal and valence during the competitive task and that the other half 
of the listeners understood their partners' valence during the cooperative task. With significantly positive correlation coefficients in the competitive task, the mean correlation coefficients of each emotional state were not strong $(0.41$ $(S D=0.14)$ for arousal, $0.44(S D=0.14)$ for valence, and $0.43(S D=0.12$ ) for positivity). With significantly positive correlation coefficients in the cooperative task, the mean correlation coefficients of each emotional state were also not strong $(0.42(S D=0.11)$ for arousal, $0.46(S D=0.14)$ for valence, and 0.44 ( $S D=0.14)$ for positivity, respectively). These results suggest that the listeners who showed a significantly positive correlation did not understand the speakers' emotion accurately but understood it moderately.

4.2. Actual and believed emotional synchrony

As Table 2 shows, only one half of the listeners moderately understood their partners' arousal in the competitive task and their valence in both tasks. Therefore, the emotional synchrony between them might be based on the inaccurate understanding of emotion.

To measure emotional synchrony with the emotional state evaluation, each pair's correlation coefficients of each emotional state through the dialogs were calculated. Positive correlation coefficients imply that their emotion was synchronized through the dialog. On the contrary, negative correlation coefficients imply that their emotion was asynchronized. With the two types of evaluated scores (selfevaluated scores and scores evaluated by their listeners), two types of correlation coefficients were calculated: the correlation coefficients between Speaker A's (B's) self-evaluated emotion and Speaker B's (A's) emotion evaluated by Speaker A (B; SI) and the correlation coefficients between Speaker A's self-evaluated emotion and Speaker B's self-evaluated emotion (SS). The SI correlation coefficient indicates the believed emotional synchrony between the speaker's own emotion and his/her partner's emotion evaluated by him/her. The SS correlation coefficient indicates the actual emotional synchrony of the speaker's own internal emotion within each pair. With these SI and SS correlation coefficients, the difference between the actual emotional synchrony and the believed emotional synchrony was investigated.

A $2 \times 2$ analysis of variance (ANOVA) for each pair's correlation coefficients of the evaluation of each emotion was performed with the factors of task (competitive and cooperative) and type of synchrony (SI and SS). Figure 2 shows the result of ANOVA for each emotional state. The results revealed a significant main effect of type of synchrony $(F(1,25)=4.40, \quad p<0.05)$ and a marginally significant interaction between the task and type of synchrony $(F(1,77)=3.40, p=0.07)$ for arousal (Fig. 2 left). For valence (Fig. 2 middle), both the task and type of synchrony showed a significant main effect $(F(1,25)=19.78, p<$ $0.001, F(1,25)=20.27, p<0.001$, respectively). Moreover, the result of ANOVA for positivity (Fig. 2 right) revealed a significant main effect of task $(F(1,25)=5.64, p<0.05)$ and type of synchrony $(F(1,25)=7.04, p<0.05)$ and a marginally significant interaction between them $(F(1,77)=3.63$, $p=0.06)$.

For all emotional states, there were significant main effects of the type of synchrony: The believed emotional

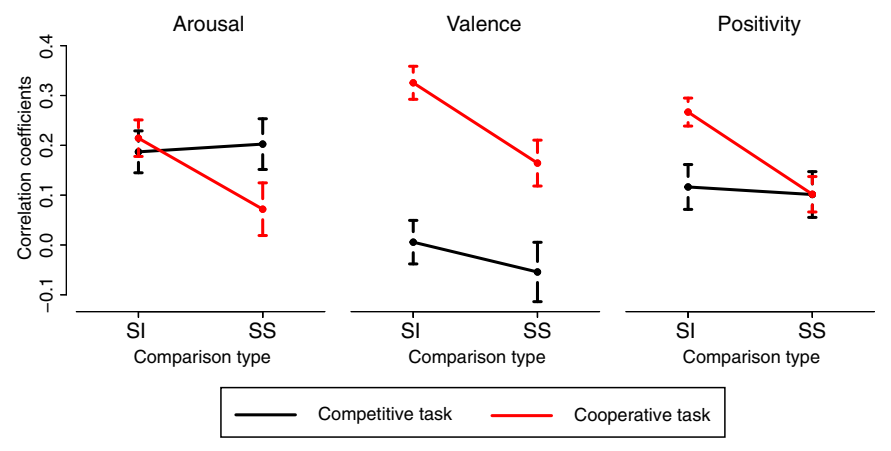

Fig. 2 Result of task $\times$ comparison type ANOVA for correlate coefficients. Error bars denote the standard errors.

synchrony (SI) showed a higher correlation than did the actual emotional synchrony (SS). This finding suggests that the pairs continued to talk with each other believing that they were more emotionally synchronized than they actually were. Moreover, there were marginally significant interactions between the task and the types of synchrony for arousal and positivity, and the believed emotional synchrony (SI) showed a higher correlation during the cooperative task. These findings suggest that they more strongly believed that they were emotionally synchronized in the cooperative task than in the competitive task because they had to cooperate with each other to solve the problem in the cooperative task.

\section{Conclusion}

The present study investigated (1) how accurately a listener understood a speaker's emotion and (2) whether the believed emotional synchrony was observed more often than actual emotional synchrony due to the listener's inaccurate emotional understanding of the speaker. Two types of taskoriented dyadic dialogs (a competitive task and a cooperative task) were recorded, and subjective evaluations of the speakers' dynamic emotional state were conducted.

The results of the correlation tests between the selfevaluation and the listeners' evaluation of the speakers' emotion revealed that the mean correlation coefficients among the pairs were low. This finding implies that the listeners did not understand the speakers' emotion accurately during the competitive task or the cooperative task dialogs. Moreover, the result of the $2 \times 2$ analysis of variance of each pair's correlation coefficients of the evaluation of each emotion with the factors of task (competitive and cooperative) and type of synchrony (the believed and the actual) revealed significant main effects of the type of synchrony and showed marginally significant interactions between the task and the types of synchrony for arousal and positivity. This finding suggests that listeners and speakers continued to talk with each other with the belief that they were more emotionally synchronized than they actually were and that this belief was stronger in the cooperative task than in the competitive task.

The interesting points of this study are that the results elucidate two peculiar facts of human nature regarding emotional communication. One is that we keep talking with each other without knowing each other's actual emotional 
state. The second is that we believe ourselves to be emotionally synchronized with our partner when we have to be cooperative with each other.

Information technology has made huge efforts to create an emotion recognition system to facilitate human-to-computer emotion communication or computer-aided emotion communication. However, our emotion communication might not be based on the proposition that we mutually and accurately understand our emotion when talking. The above results would give a novel perspective to approach the hard task of facilitating human-to-computer emotion communication or computer-aided emotion communication.

A further investigation should be performed on different communication environments, i.e., a non-face-to-face environment, where communication channels between the speakers are regulated. Such an investigation could elucidate the differences between face-to-face communication and nonface-to-face communication and determine the key channel for people to communicate their emotion for a better understanding of each other's emotion.

\section{Acknowledgements}

The contents of this report were presented at the Fall Meeting of ASJ in 2013.

\section{References}

[1] H. H. Clark, "Communities, commonalities and communication," in Rethinking Linguistic Relativity, J. J. Gumperz and S. C. Levinson, Eds. (Cambridge University Press, Cambridge, 1996), pp. 324-355.

[2] W. Ickes, L. Stinson, V. Bissonnette and S. Garcia, "Naturalistic social cognition: Empathic accuracy in mixed-sex dyads," J. Pers. Soc. Psychol., 59, 730-742 (1990).
[3] L. Stinson and W. Ickes, "Empathic accuracy in the interactions of male friends versus male strangers," J. Pers. Soc. Psychol., 62, 787-797 (1992).

[4] L. L. Verhofstadt, A. Buysse, W. Ickes, M. Davis and I. Devoldre, "Support provision in marriage: the role of emotional similarity and empathic accuracy," Emotion, 8, 792-802 (2008).

[5] J. Zaki, N. Bolger and K. Ochsner, "It takes two: the interpersonal nature of empathic accuracy," Psychol. Sci., 19, 399-404 (2008).

[6] J. Decety and W. Ickes, The Social Neuroscience of Empathy (The MIT Press, Cambridge, Mass., 2011).

[7] U. Hess and S. Blairy, "Facial mimicry and emotional contagion to dynamic emotional facial expressions and their influence on decoding accuracy," Int. J. Psychophysiol., 40, 129-141 (2001).

[8] C. K. Hsee, E. Hatfield and C. Chemtob, "Assessments of the emotional states of others: Conscious judgments versus emotional contagion," J. Soc. Clin. Psychol., 11, 119-128 (2011).

[9] G. Dezecache, L. Conty, M. Chadwick, L. Philip, R. Soussignan, D. Sperber and J. Grèzes, "Evidence for unintentional emotional contagion beyond dyads," PloS one, 8 , e67371 (2013).

[10] R. Cowie, "Perceiving emotion: towards a realistic understanding of the task," Philos. Trans. R. Soc. Lond. Ser. B Bio. Sci., 364, 3515-3525 (2009).

[11] Y. Arimoto, H. Kawatsu, S. Ohno and H. Iida, "Naturalistic emotional speech collection paradigm with online game and its psychological and acoustical assessment," Acoust. Sci. \& Tech., 33, 359-369 (2012).

[12] K. P. Truong, D. A. van Leeuwen and F. M. de Jong, "Speechbased recognition of self-reported and observed emotion in a dimensional space," Speech Commun., 54, 1049-1063 (2012).

[13] R. Cowie, G. McKeown and E. Douglas-Cowie, "Tracing emotion," Int. J. Synth. Emotions, 3, 1-17 (2012). 